\title{
TRIB2 knockdown as a regulator of chemotherapy resistance and proliferation via the ERK/STAT3 signaling pathway in human chronic myelogenous leukemia K562/ADM cells
}

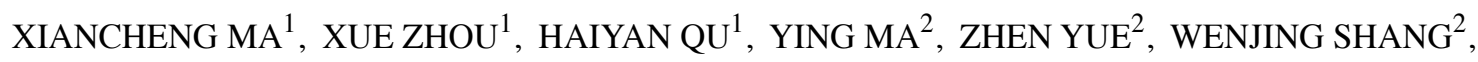 \\ PINGYU WANG ${ }^{2}$, SHUYANG XIE ${ }^{2}$, YOUJIE LI $^{2}$ and YUNXIAO SUN ${ }^{1}$ \\ ${ }^{1}$ Department of Pediatrics, Yantai Affiliated Hospital of Binzhou Medical University, Yantai, Shandong 264100; \\ ${ }^{2}$ Key Laboratory of Tumor Molecular Biology in Binzhou Medical University, \\ Department of Biochemistry and Molecular Biology, Binzhou Medical University, Yantai, Shandong 264003, P.R. China
}

Received September 26, 2017; Accepted January 31, 2018

DOI: 10.3892/or.2018.6249

\begin{abstract}
Acquired resistance to chemotherapy plays a critical role in human drug treatment failure in many tumor types. Multidrug resistance (MDR) to Adriamycin (ADM) also limits the efficacy of therapy in human chronic myelogenous leukemia (CML). The overexpression of drug efflux transporters is one mechanism uderlying MDR. In particular, the consistent activation of MDR1 and MDR-associated protein 1 (MRP1) is involved in drug resistance. In the present study, ADM-resistant human CML K562/ADM cells were stably transfected with a Tribbles homolog 2 (TRIB2)-targeted vector. A CCK-8 assay showed that the half maximal inhibitory concentration $\left(\mathrm{IC}_{50}\right)$ of $\mathrm{ADM}$ and the cell proliferation were lower in the transfected cells compared with that in the parental K562/ADM cells. The mRNA and protein expression levels of MDR1 and MRP1 were determined by reverse transcription-polymerase chain reaction (RT-PCR), RT-quantitative PCR and western blot analysis. The results showed that the expression of MDR1 and MRP1
\end{abstract}

Correspondence to: Dr Yunxiao Sun, Department of Pediatrics, Yantai Affiliated Hospital of Binzhou Medical University, 717 Jinbu Street, Yantai, Shandong 256003, P.R. China

E-mail: sunyunxiao1979@163.com

Dr Youjie Li, Key Laboratory of Tumor Molecular Biology in Binzhou Medical University, Department of Biochemistry and Molecular Biology, Binzhou Medical University, 346 Guanhai Road, Yantai 264003, P.R. China

E-mail: youjie1979@163.com

Abbreviations: CML, chronic myeloid leukemia; CCK-8, Cell Counting Kit-8; GFP, green fluorescent protein; RT-qPCR, reverse transcription-quantitative polymerase chain reaction; SDS-PAGE, sodium dodecyl sulphate-polyacrylamide gel electrophoresis; TRIB2, tribble homologue 2; MDR1, multidrug resistance 1; MRP1, multidrug resistance-associated protein 1

Key words: TRIB2, adriamycin resistance, proliferation, MDR1, MRP1 was significantly reduced in K562/ADM cells transfected with pGPU6/GFP/Neo-TRIB2. Due to the downregulation of MDR1 and MRP1, the intracellular accumulation of ADM was increased in the transfected cells compared with that in the parental K562/ADM cells. Therefore, the sensitivity of the K562/ADM cells to ADM was enhanced and proliferation was inhibited. Our research revealed that protein expression of the ERK signaling pathway was inhibited by downregulating TRIB2, indicating that the ERK pathway was involved in cell drug resistance and proliferation. Furthermore, we used the ERK-specific blocker U0126 to demonstrate this phenomenon. In summary, our research suggested that knockdown of TRIB2 could slow cell growth and reverse resistance, implying that TRIB2 is a potential therapy target for resistant human CML.

\section{Introduction}

Chronic myeloid leukemia (CML) is one of the most prevalent types of myeloproliferative neoplasm, and its multidrug resistance (MDR) is usually associated with a poor clinical outcome (1,2). MDR1 and MDR-associated protein 1 (MRP1) belong to the protein family of ATP-binding cassette transporters, which use the energy released by ATP hydrolysis to bind drugs and export them from the cell $(3,4)$. Adriamycin-resistant CML K562 (K562/ADM) cells reportedly overexpress MDR1 and MRP1 (5), meaning that the resistance of this cell line is associated with abnormalities in drug efflux. In the present study, we found that the proliferation of K562/ADM cells was significantly inhibited upon knockdown of the Tribbles homolog 2 (TRIB2) gene, compared with that noted in the untreated cells. The reason for this growth inhibition in the resistant cell line may be associated with cell drug-resistance reversal. It is known that TRIB2 is expressed in mammals. TRIB protein family members encode pseudo-kinase proteins that are highly conserved in evolution, and act as adaptors in signaling pathways for important cellular processes $(6,7)$. Previous studies have focused on the pathological role of TRIB2 in various diseases, including CML, and metabolic and neurological diseases, in which it has been identified as a critical signaling modulator and mediator $(8,9)$. Related reports 
suggest that TRIB2 overexpression can indeed promote tumor resistance by activating relevant cell pathways (10). However, little is known concerning the effects of TRIB2 gene knockdown on drug-resistant proteins.

Previous studies have found that the downregulation of drug-resistance proteins may partly depend on inhibition of the ERK pathway in cancer (11-13). In the present study, we explored changes in the ERK signaling pathway after knockdown of the TRIB2 gene in K562/ADM cells. The aim of the present study was to explore the effect of the downregulation of TRIB2 expression on the chemotherapy resistance and proliferation of K562/ADM cells. The results provide a novel basis for the treatment of clinical drug resistance mechanisms, and potential routes for therapeutic strategies in CML.

\section{Materials and methods}

Cell lines and cell culture. Human CML K562 cells and the MDR sub-cell line K562/ADM cells were obtained from the Institute of Medical Molecular Genetics of Binzhou Medical University (Yantai, China). The cells were maintained in RPMI-1640 basic medium (1X) supplemented with $10 \%$ fetal bovine serum (FBS; both from Gibco; Thermo Fisher Scientific, Inc., Waltham, MA, USA) at $37^{\circ} \mathrm{C}$ in a humidified atmosphere containing $5 \% \mathrm{CO}_{2}$. K562/ADM cells were maintained in the presence of $7 \mu \mathrm{M}$ ADM (Wanle, Shenzhen, China). Prior to the experiment, the cells were cultured in drug-free medium for 1 week. In addition, K562/ADM and K562/ADM-TRIB2 cells were pretreated for $24 \mathrm{~h}$ with $10 \mu \mathrm{M}$ U0126 (Shanghai Selleck Chemicals Co., Ltd., Shanghai, China) in order to study the ERK pathway.

Cell transfection. A pair of vector sequences targeting TRIB2 were generated and named pGPU6/GFP/Neo-shNC, pGPU6/GFP/Neo-TRIB2 and pGPU6/GFP/Neo-TRIB2-1. Firstly, cells were seeded at a density of $0.9-4 \times 10^{5} /$ well into a 6-well plate with $1.6 \mathrm{ml}$ 10\% FBS-containing RPMI-1640 medium the day before transfection. After incubation for $24 \mathrm{~h}$, cell confluence was $40-80 \%$ on the day of transfection. DNA $(0.4 \mu \mathrm{g})$ was dissolved in TE buffer and added to $3.2 \mu \mathrm{l}$ Enhancer; $10 \mu \mathrm{l}$ Effectene Transfection reagent (Qiagen China Co., Ltd., Shanghai, China) was added after 5 min of incubation. After incubating for a further 6-18 h, the medium was removed and replaced with fresh medium. Transfection efficiency was determined using fluorescence microscopy and the non-transfected K562/ADM cells were used as the control. After $48 \mathrm{~h}$ of transfection, G418 (500 ng/ml; Thermo Fisher Scientific, Inc.) was added to the medium. Stable positive clones were obtained after 4 weeks of selection. Administration of G418 was halted at 2 weeks prior to the experiment.

Cell proliferation assay. K562/ADM cells $\left(8 \times 10^{3}\right)$ with or without knockdown of TRIB2 were divided into $0,24,48$ and $72 \mathrm{~h}$ experimental groups, seeded into 96-well plates and incubated with medium containing 10\% FBS. Each group consisted of five parallel wells, with parental K562/ADM cells serving as the control. Then, $10 \mu \mathrm{l}$ CCK-8 (Dojindo Molecular Technologies, Inc., Shanghai, China) solution was added to each well and incubated for a further $4 \mathrm{~h}$. Then, the absorbance at $450 \mathrm{~nm}$ was measured using a fluorescence spectrophotometer (F-7000; Hitachi, Ltd., Tokyo, Japan). The absorbance values were collected for processing using GraphPad Prism 5 software (GraphPad Software, Inc., La Jolla, CA, USA).

CCK-8 analysis of the half maximal inhibitory concentration $\left(I C_{50}\right)$. The CCK-8 was used to determine the inhibition ratio of cells incubated with various concentrations of ADM (0-16 $\mu \mathrm{M}$ for the K562 cells; 15-120 $\mu \mathrm{M}$ for the K562/ADM cells, K562/ADM-Con cells or K562/ADM-TRIB2 cells). After dilution in RPMI-1640 medium for $24 \mathrm{~h}, 10 \mu \mathrm{l} \mathrm{CCK}-8$ solution was added to each well and incubated for $4 \mathrm{~h}$. The absorbance was then measured at $450 \mathrm{~nm}$ with a microplate reader. A blank well containing only medium and ADM was used as a control. The concentration of $\mathrm{ADM}$ that resulted in the $\mathrm{IC}_{50}$ was calculated. Resistant fold= $\mathrm{IC}_{50} \mathrm{~K} 562 / \mathrm{ADM} / \mathrm{IC}_{50} \mathrm{~K} 562$. Reversal fold $=\mathrm{IC}_{50}$ K562/ADM-TRIB2 group/ $\mathrm{IC}_{50}$ control.

Flow cytometry. K562, K562/ADM, and cells transfected with pGPU6/GFP/Neo-TRIB2 and pGPU6/GFP/Neo-shNC were seeded into a 6 -well plate at a density of $5 \times 10^{5}$ cells/well. The non-transfected group was defined as the blank control. A total of $5 \mu \mathrm{M}$ ADM was applied to the wells. After incubation for $1 \mathrm{~h}$, the cells were harvested via centrifugation and washed twice with ice-cold PBS. The cell-associated mean fluorescence intensity (MFI) of ADM was detected using a FACSCalibur flow cytometer (FACS FC500; Beckman Coulter, Inc., Brea, CA, USA), with excitation/emission wavelengths of $485 / 580 \mathrm{~nm}$.

Reverse transcription-polymerase chain reaction (RT-PCR) and $R T$-quantitative PCR (RT-qPCR). According to the manufacturer's instructions, total RNA was isolated using TRIzol reagent (Thermo Fisher Scientific, Inc.) and assessed at a ratio of A260/A280 by spectrophotometry (Nano Drop 2000; Nano Drop Technologies, Inc., Wilmington, DE, USA). RNA (1-2 $\mu \mathrm{g})$ was used to synthesize the first-strand cDNA. The primers used in this experiment were designed and synthesized by Shanghai GenePharma, Co., Ltd. (Shanghai, China) and are presented in Table I. Prime Script ${ }^{\mathrm{TM}}$ RT reagent kit with gDNA Eraser (Takara Bio, Inc., Otsu, Japan) was used to perform the RT reaction. Then, Premix Taq ${ }^{\mathrm{TM}}$ (Takara Bio, Inc.) was used to perform PCR amplification on the Eppendorf Mastercycler Personal system (Eppendorf China Ltd., Hong Kong, China). The reaction system contained forward primer, reverse primer, Premix Taq and template cDNA. The PCR products were separated on $1 \%$ agarose gels (Takara Bio, Inc.), stained with G-Red nucleic acid dyes (1:10,000; BioTeke Corporation, Beijing, China). The images were captured with a Tanon gel imaging system (Tanon, Shanghai, China). The parental non-transfected K562/ADM cells were used as the blank control. GAPDH served as an internal standard for quality control and quantification of target genes.

RT-qPCR was performed with a Step One ${ }^{\mathrm{TM}}$ Real-Time PCR system (Applied Biosystems; Thermo Fisher Scientific, Inc.) and SYBR Premix Ex Taq ${ }^{\mathrm{TM}}$ (Takara Bio, Inc.). The reaction system of PCR contained SYBR Premix Ex Taq ${ }^{\mathrm{TM}}$, the forward primer, the reverse primer, template cDNA and nuclease-free distilled water. The results were calculated using the $2^{-\Delta \Delta \mathrm{Cq}}$ value.

Western blot analysis. Cells cultured in 6-well plates were harvested and washed with cold PBS three times. A total of 
Table I. Primers used in reverse transcription-quantitative polymerase chain reaction.

\begin{tabular}{llc}
\hline Gene & \multicolumn{1}{c}{ Primer sequence } & Product length (bp) \\
\hline MDR1 & Forward: 5'-GGAGCCTACTTGGTGGCACATAA-3' & 121 \\
& Reverse: 5'-TGGCATAGTCAGGAGCAAATGAAC-3' & 133 \\
MRP1 & Forward: 5'-CAGCCCTTCCTGACAAGCTA-3' \\
& Reverse: 5'-GTGGCCTCATCCAACACAAG-3' \\
TRIB2 & Forward: 5'-CTCCGAACTTGTCGCATTG-3' \\
& Reverse: 5'-CACATAGGCTTTGGTCTCAC-3' \\
GAPDH & Forward: 5'-CAGCCCTTCCTGACAAGCTA-3' & 233 \\
& Reverse: 5'-GTGGCCTCATCCAACACAAG-3' & 133 \\
\hline
\end{tabular}

MDR1, multidrug resistance 1; MRP1, MDR-associated protein 1; TRIB2, Tribble homologue 2.

Table II. $\mathrm{IC}_{50}$ values for $\mathrm{K} 562 / \mathrm{ADM}$ cells and $\mathrm{K} 562$ cells toward ADM by CCK-8 assay (means $\pm \mathrm{SD} ; \mathrm{n}=5$ ).

\begin{tabular}{lccc}
\hline & \multicolumn{2}{c}{$\mathrm{IC}_{50} \pm \mathrm{SD}(\mu \mathrm{M})$} & \\
\cline { 2 - 3 } Treatment & $\mathrm{K} 562$ & $\mathrm{~K} 562 / \mathrm{ADM}$ & $\mathrm{RF}$ \\
\hline Adriamycin & $3.219 \pm 0.921$ & $84.801 \pm 0.0183^{\mathrm{a}}$ & 26.22
\end{tabular}

${ }^{\text {aP }}<0.01$ vs. K562 group. RF, Resistant fold; ADM, Adriamycin.

$120 \mu$ l RIPA lysis buffer (Beyotime Institute of Biotechnology, Shanghai, China) was added to extract the proteins. Protein samples were separated via 10 or $6 \%$ SDS-PAGE (Beyotime Institute of Biotechnology) with a constant voltage of $80 \mathrm{~V}$ for $0.5 \mathrm{~h}$, which was then switched to $120 \mathrm{~V}$ for a further $1 \mathrm{~h}$. Proteins were then transferred onto polyvinylidine difluoride membranes (EMD Millipore, Billerica, MA, USA). The membranes were blocked with $5 \%$ skimmed dry milk in $1 \mathrm{X}$ Tris-buffered saline with Tween-20 (pH 8.0) at room temperature for $2 \mathrm{~h}$, and then incubated overnight at $4^{\circ} \mathrm{C}$ with six styles of primary antibodies respectively. The primary antibodies were rabbit polyclonal anti-TRIB2 (1:500; cat. no. 204119; Abcam, Cambridge, UK), anti-MDR1 (1:500; cat no. 0563R; BIOSS, Beijing, China), anti-MRP1 (1:500; cat. no. 0657R; BIOSS), anti-STAT3 (1:500; cat. no. 1141R; Bioworld Technology, Inc., Nanjing, China), anti-p-ERK (1:500, cat. no. 5016; Bioworld Technology, Inc.) and anti-GAPDH (1:1,000; cat. no. AP0063; Bioworld Technology, Inc.). Following this, the membranes were incubated with a horseradish peroxidase-conjugated goat anti-rabbit immunoglobulin G (1:5,000; cat no. 13278; Bioworld Technology, Inc.) for 2 h. Finally, images were captured using a FluorChem FC2 gel imaging system (Protein Simple, San Jose, CA, USA). The intensity of each band was normalized to GAPDH in the respective lane, and the K562/ADM cells were as control.

Data analysis. Statistical analyses were performed using SPSS 21.0 software (IBM Corp., Armonk, NY, USA). Independent two-sample t-tests were used to analyze differences between two groups. One-way analysis of variance (ANOVA) was used to analyze differences among three or more groups.
Table III. Construction of the expression vector and its interference sequence $\left(5^{\prime}-3^{\prime}\right)$.

\begin{tabular}{lc}
\hline Vector construction & \multicolumn{1}{c}{ Interference sequence } \\
\hline pGPU6/GFP/Neo-shNC & GTTCTCCGAACGTGTCACGT \\
pGPU6/GFP/Neo-TRIB2 & TAGCGAGATATGGGAGATC \\
pGPU6/GFP/Neo-TRIB2-1 & CTTGTCGCATTGCGTTTCTTG
\end{tabular}

A post-hoc test of ANOVA was conducted by performing a Tukey's test. Data are expressed as the mean \pm standard deviation. Statistical significance was accepted at $\mathrm{P}<0.05$.

\section{Results}

Calculation of the level of ADM drug-resistance in the K562 and K562/ADM cell groups. The $\mathrm{IC}_{50}$ was calculated by performing a CCK-8 spectrophotometric assay. The data from the K562/ADM cells were markedly higher compared with K562 cells, with a resistance ratio of 26.22 for K562/ADM to K562 cells (Table II). There was a significant difference in intracellular ADM accumulation between the K562/ADM group and the K562 group (Fig. 1). All values were statistically significant $(\mathrm{P}<0.01)$.

Determination of relative protein expression in $K 562$ and K562/ADM cells. We evaluated MDR1, MRP1 and TRIB2 expression in the non-resistant K562 cells and the ADM-resistant K562/ADM cells. The results indicated that the K562 and K562/ADM cells both showed high expression levels of all three proteins. The K562/ADM cells exhibited higher mRNA expression of TRIB2, MDR1 and MRP1, compared with the K562 cells (Fig. 2A and B). Western blot analyses revealed the same expression trends at the protein level (Fig. 2C and D).

Construction of a stable TRIB2 transfection cell system. Toevaluate the functional changes in the K562/ADM cells following silencing of TRIB2, we transfected pGPU6/GFP/Neo-shNC, pGPU6/GFP/Neo-TRIB2 and pGPU6/GFP/Neo-TRIB2-1 (Table III) into K562/ADM cells, with untreated K562/ADM 
A

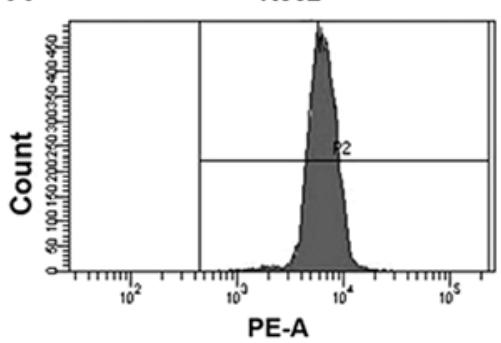

B

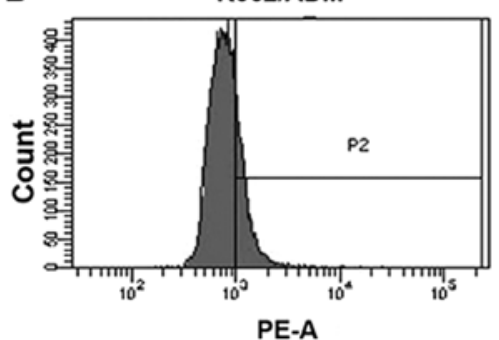

C

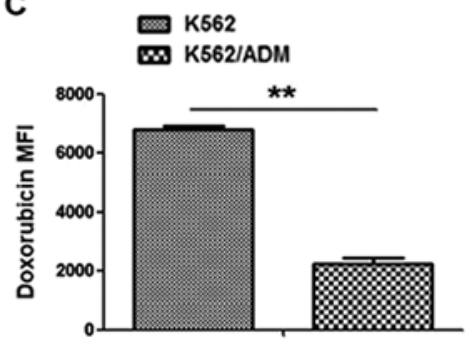

Figure 1. Determination of intracellular Adriamycin accumulation was assessed by flow cytometry. (A) Intracellular accumulation of Adriamycin was examined in K562 cells. (B) Intracellular accumulation of Adriamycin in K562/ADM was significantly lower compared with that in the K562 cells. (C) Comparison of doxorubicin (Adriamycin) MFI in K562 and K562/ADM cells. All data are presented as the mean \pm standard deviation ( $\mathrm{n}=3$ ). ${ }^{* *} \mathrm{P}<0.01$.

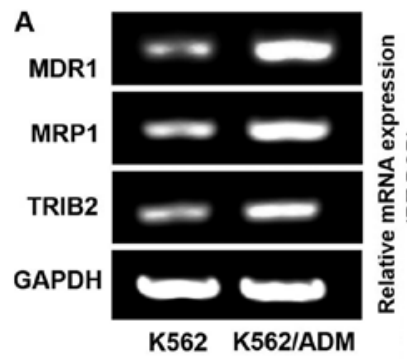

C

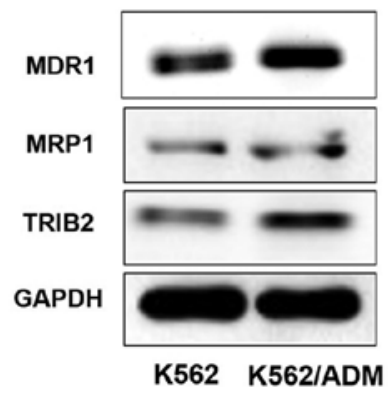

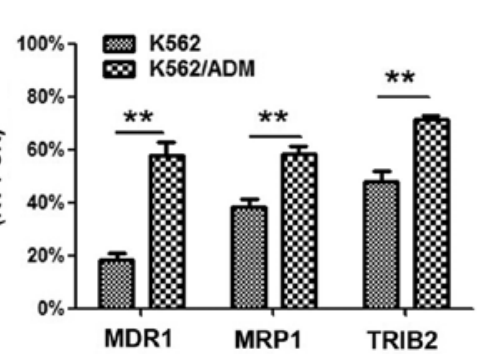

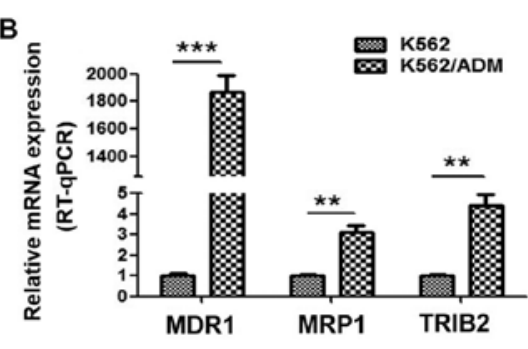

D

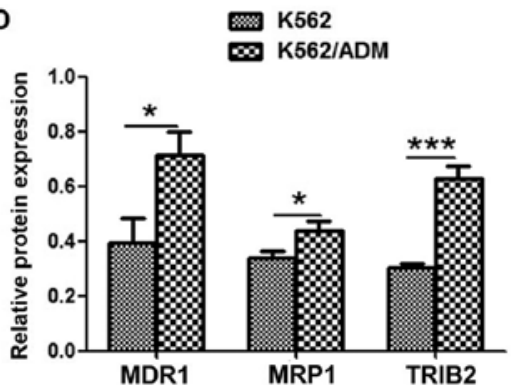

Figure 2. Comparison of MDR1, MRP1 and TRIB2 gene expression. (A) RT-PCR indicated that MDR1, MRP1 and TRIB2 expression was lower in K562 cells than in K562/ADM cells. (B) The $2^{-\Delta \Delta C q}$ values were detected by qPCR. (C) Protein expression of the three target genes in K562/ADM cells was higher than that in the K562 cells. (D) Optical density results indicating protein expression. GAPDH was used as an internal reference. All data are presented as the mean \pm standard deviation $(\mathrm{n}=3) .{ }^{*} \mathrm{P}<0.05,{ }^{* *} \mathrm{P}<0.01$ and ${ }^{* * *} \mathrm{P}<0.001$. MDR1, multidrug resistance 1; MRP1, multidrug resistance-associated protein 1; TRIB2, Tribble homologue 2.

cells serving as the control. Green fluorescence is only detected in cells successfully transfected with GFP. Therefore, cells with green fluorescence indicate a high efficiency of transfection. After 4 weeks of G418 selection, we successfully obtained stable positive clones (Fig. 3A). RT-PCR, RT-qPCR and western blot analyses revealed that TRIB2 expression was markedly decreased in the K562/ADM-TRIB2 group and the K562/ADM-TRIB2-1 group at the transcription and translation levels, compared with that in the K562/ADM group. TRIB2 expression in the K562/ADM-Con group was not significantly different from the negative control group (Fig. 4).

TRIB2 knockdown inhibits cell proliferation. A CCK-8 assay was performed to evaluate cell proliferation (Fig. 3B). According to the growth curve, we found that the proliferation of cells treated with pGPU6/GFP/Neo-TRIB2 and pGPU6/GFP/Neo-TRIB2-1 was markedly inhibited, while cells transfected with pGPU6/GFP/Neo-shNC exhibited no significant difference. The results also showed that pGPU6/GFP/Neo-TRIB2 was more effective than
pGPU6/GFP/Neo-TRIB2-1. Then, pGPU6/GFP/Neo-TRIB2 was used to explore the effect of the downregulation of TRIB2 expression on the chemotherapy resistance and proliferation of K562/ADM cells.

TRIB2 knockdown decreases $I C_{50}$ and increases intracellular ADM accumulation. The $\mathrm{IC}_{50}$ value was calculated by performing a CCK- 8 spectrophotometric assay. The $\mathrm{IC}_{50}$ of the K562/ADM-Con group was not observed to be significantly different compared with the K562/ADM cells, while a reduction in the $\mathrm{IC}_{50}$ value was obvious in the K562/ADM-TRIB2 group, with a reversal fold of 12.12 (Table IV). The intracellular ADM accumulation in K562/ADM-TRIB2 cells was considerably higher than that in the K562/ADM cells and K562/ADM-Con cells (Fig. 5). All values were statistically significant $(\mathrm{P}<0.01)$.

Decreased expression of MDRI and MRPI by TRIB2 knockdown. MDR1 and MRP1 are ABC transporters overexpressed in many drug-resistant tumor cells, which contribute 

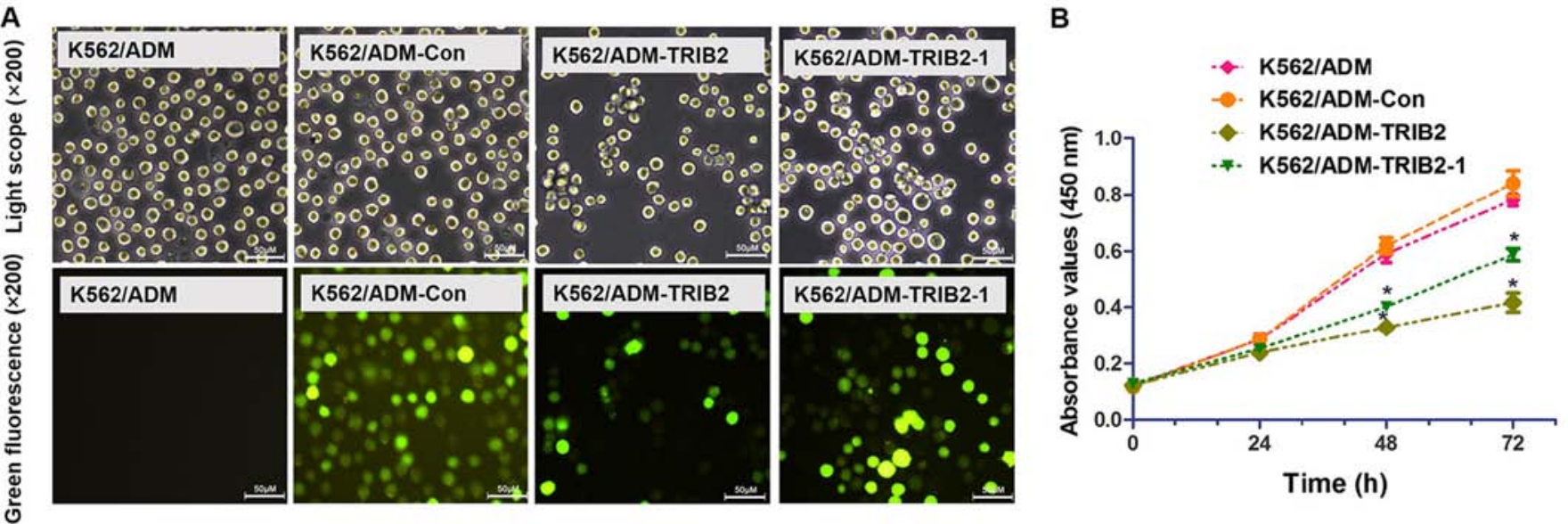

Figure 3. Determination of transfection efficiency and cell proliferation. (A) Transfection efficiency was detected by fluorescence microscopy. The average transfection efficiency of each group was $>90 \%$ after 4 weeks of G418 filtration. (B) A CCK-8 assay demonstrated that the proliferation of K562/ADM-TRIB2 cells was significantly lower than the other three groups. K562/ADM-TRIB2-1 cell proliferation was lower than the parental K562/ADM cells and K562/ADM-Con cells. No significant difference was observed between K562/ADM and K562/ADM-Con cells. All data are presented as the mean \pm standard deviation ( $\mathrm{n}=3$ ). " $\mathrm{P}<0.05$ vs. the control group.

A

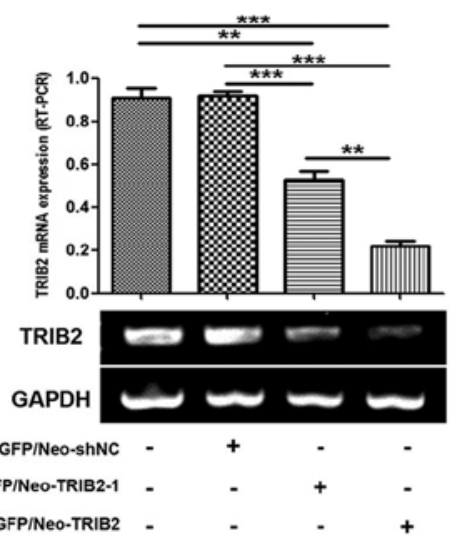

B

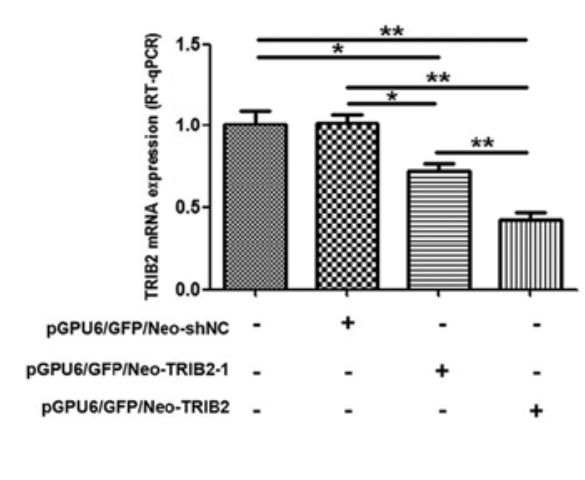

C

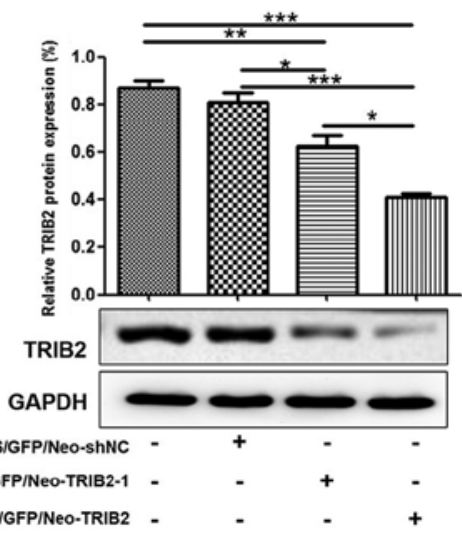

Figure 4. Detection of TRIB2 expression. (A) RT-PCR demonstrated that TRIB2 expression was significantly lower in K562/ADM-TRIB2 cells and K562/ADM-TRIB2-1 cells compared with that in the control group, and K562/ADM-TRIB2 was more effective. No significant difference was observed between the K562/ADM and K562/ADM-Con groups. (B) RT-qPCR analysis showed the same trends as RT-PCR. (C) TRIB2 protein expression was markedly reduced following the knockdown of TRIB2 in K562/ADM cells. Signal intensity was normalized to that of GAPDH. All data are presented as the mean \pm standard deviation $(\mathrm{n}=3) .{ }^{*} \mathrm{P}<0.05,{ }^{* *} \mathrm{P}<0.01$ and ${ }^{* * *} \mathrm{P}<0.001$. TRIB2, Tribble homologue 2.

Table IV. IC I0 $_{0}$ values of K562/ADM cells, K562/ADM-Con and K562/ADM-TRIB2 cells toward Adriamycin by CCK-8 assay (means \pm SD of triplicate experiments).

\begin{tabular}{lcccr}
\hline & \multicolumn{3}{c}{$\mathrm{IC}_{50} \pm \mathrm{SD}(\mu \mathrm{M})$} & $\mathrm{RF}$ \\
\cline { 2 - 5 } Treatment & $\mathrm{K} 562 / \mathrm{ADM}$ & $\mathrm{K} 562 / \mathrm{ADM}-\mathrm{Con}$ & $\mathrm{K} 562 / \mathrm{ADM}-\mathrm{TRIB} 2$ & 12.12 \\
\hline Adriamycin & $84.012 \pm 0.037$ & $75.946 \pm 0.134$ & $39.041 \pm 0.321^{\mathrm{a}}$ & \\
\hline
\end{tabular}

${ }^{\mathrm{a}} \mathrm{P}<0.01$, vs. K562/ADM and K562/ADM-Con group. RF, reversal fold; ADM, Adriamycin.

to the development of MDR. Therefore, we assessed whether TRIB2 knockdown could influence the expression of MDR1 and MRP1. Notably, western blotting, RT-PCR and RT-qPCR analyses (Fig. 6) illustrated that the expression levels of MDR1 and MRP1 were lower in the K562/ADM-TRIB2 cells, compared with levels in the control cells. This indicated that
TRIB2 may be involved in key steps of MDR development in CML.

Inhibition of the ERK pathway in K562/ADM-TRIB2 cells. The expression of p-ERK and STAT3 in K562/ADM cells was higher compared with that noted in the K562 cells. However, 
A

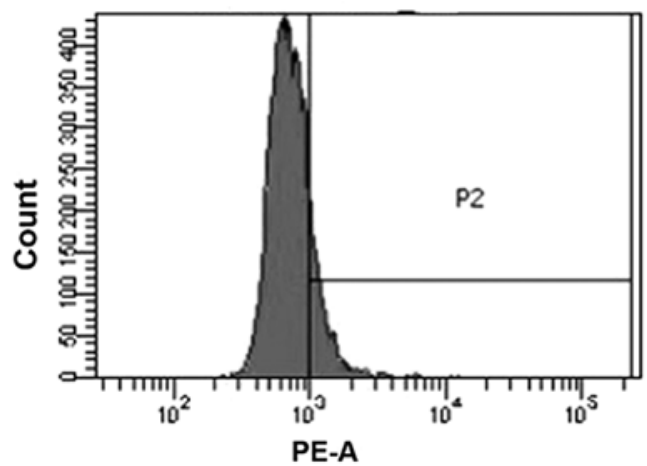

C

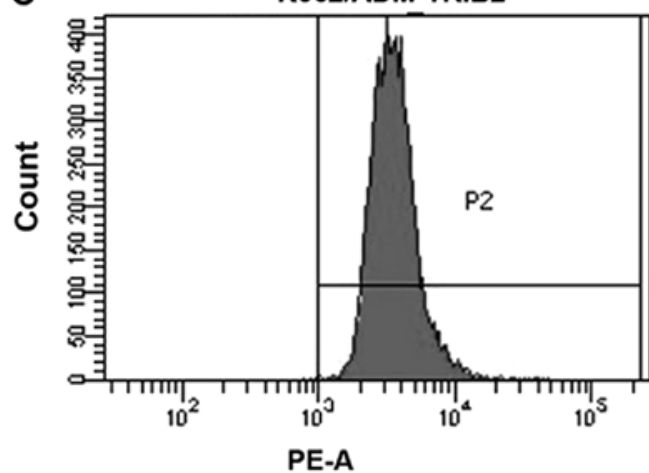

B

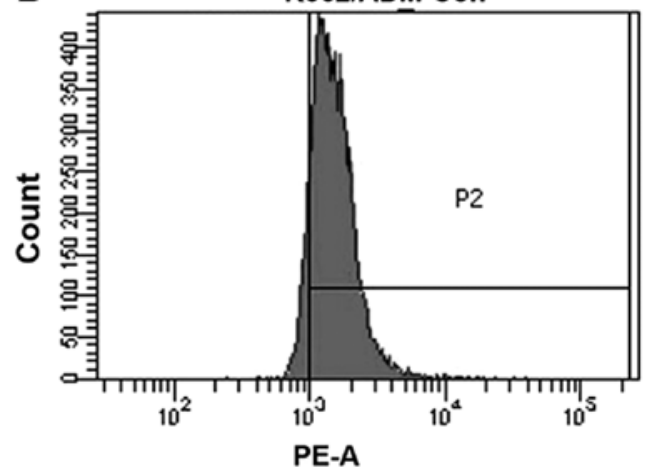

D

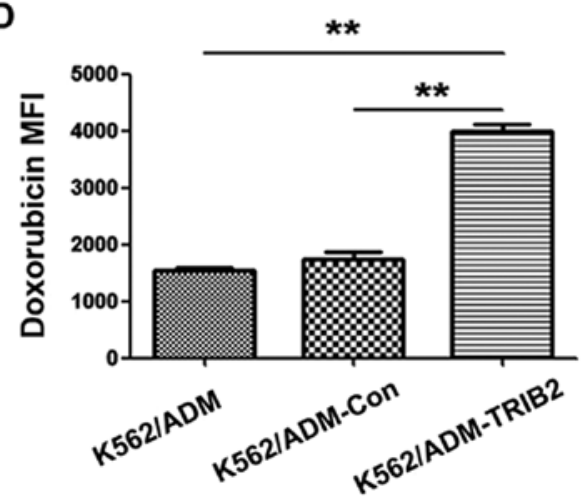

Figure 5. Analysis of intracellular Adriamycin accumulation in a stably transfected TRIB2 cell system. (A-C) No significant differences in intracellular accumulation of doxorubicin (Adriamycin) was observed between the K562/ADM-Con cells and the control group. However, the accumulation in K562/ADM-TRIB2 cells was markedly decreased. (D) ADM MFI demonstrated the differences between groups. All data are presented as the mean \pm standard deviation ( $\mathrm{n}=3$ ). ${ }^{* * *} \mathrm{P}<0.01$.

A
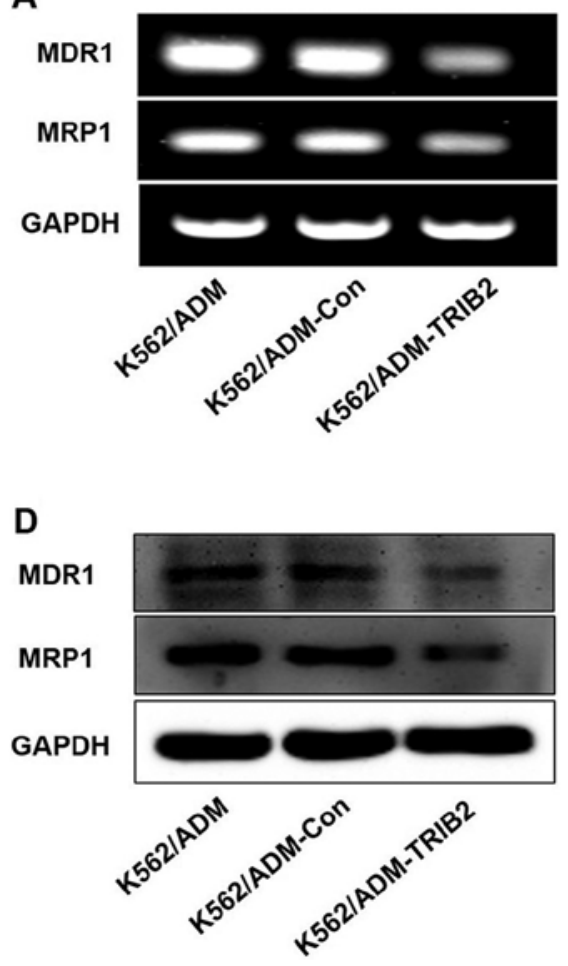
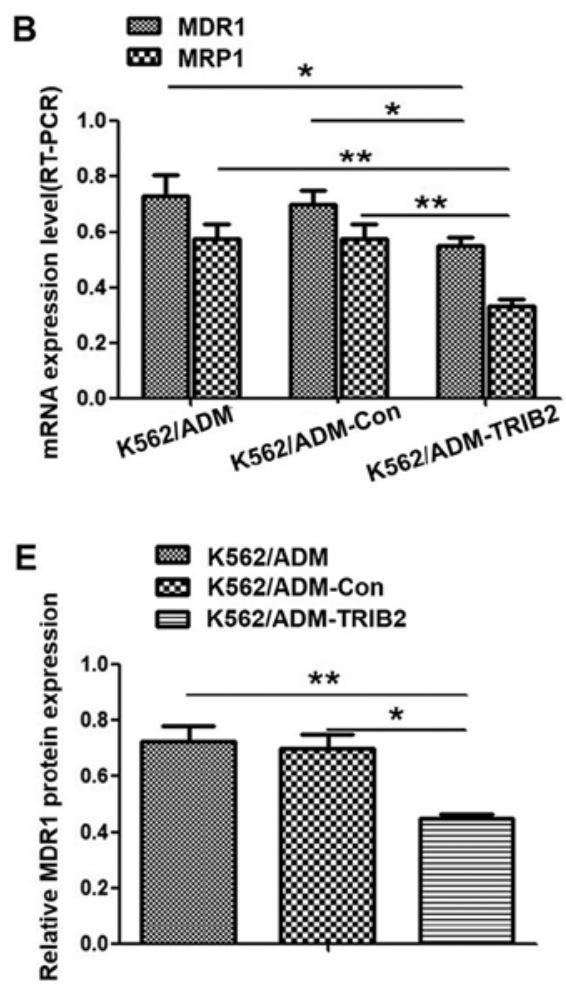

\section{C}

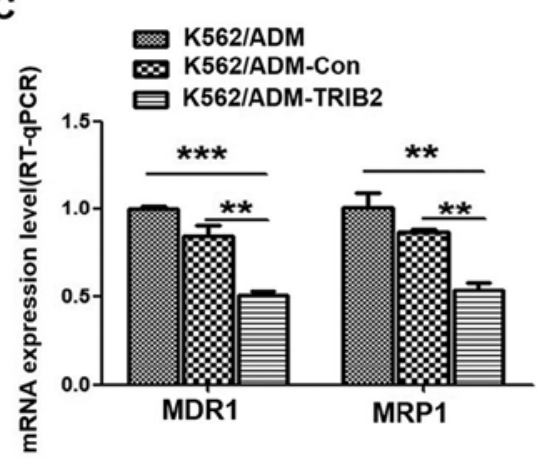

$\mathbf{F}$

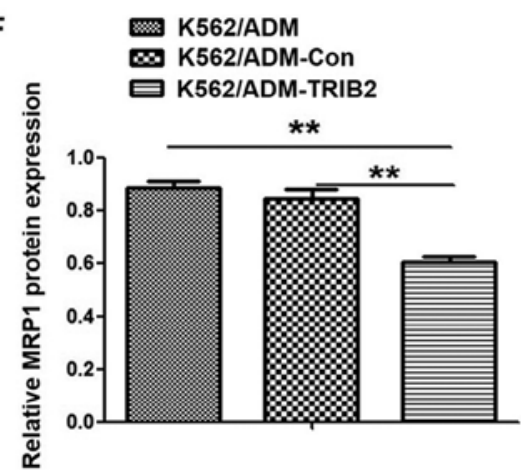

Figure 6. Analysis of MDR1 and MRP1 expression by RT-qPCR and western blotting. (A) mRNA expression of MDR1 and MRP1 was reduced significantly following TRIB2 knockdown. (B) Statistical analysis of RT-PCR optical density values. (C) Statistical analysis of RT-qPCR values. (D) MDR1 and MRP1 protein expression was lower in K562/ADM-TRIB2 cells compared with K562/ADM cells. (E) Optical density of MDR1 expression. (F) Optical density of MRP1 expression. GAPDH was used as an internal reference. Data are presented as the mean \pm standard deviation $(\mathrm{n}=3) .{ }^{*} \mathrm{P}<0.05,{ }^{* *} \mathrm{P}<0.01$ and ${ }^{* * *} \mathrm{P}<0.001$. MDR1, multidrug resistance 1; MRP1, multidrug resistance-associated protein 1; TRIB2, Tribble homologue 2. 

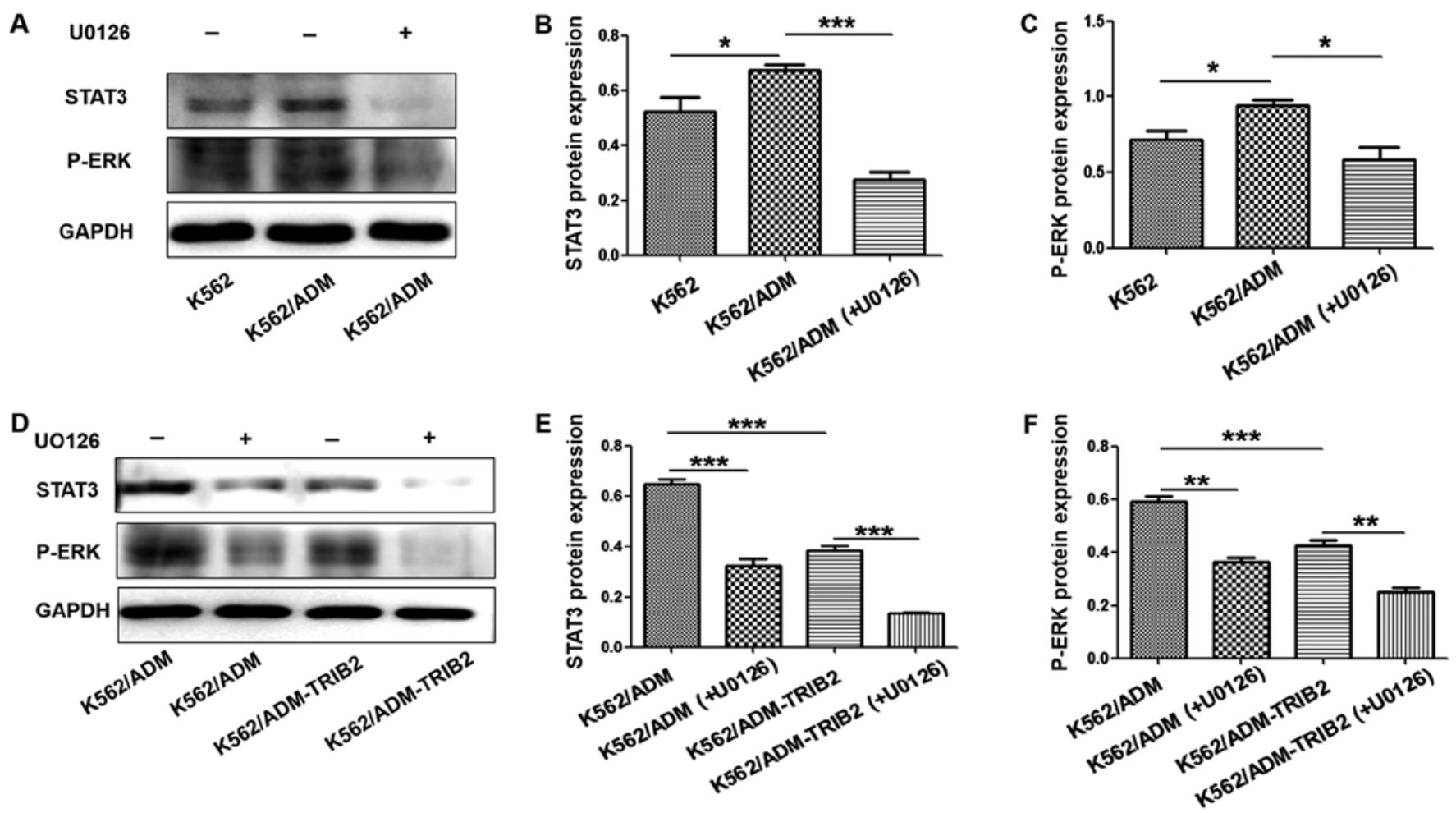

Figure 7. Changes in the ERK pathway following TRIB2 knockdown. (A) Expression of STAT3 and p-ERK in K562 cells was lower compared with that noted in the negative control cells. Expression of STAT3 and p-ERK was markedly reduced in the K562/ADM cells treated with U0126, compared with in the negative control cells. (B and C) Statistical analysis of ERK pathway protein expression. (D) Expression of STAT3 and p-ERK was lower in K562/ADM cells transfected with pGPU6/GFP/Neo-TRIB2 compared with that noted in the non-transfected group. Expression of STAT3 and p-ERK was reduced further in the K562/ADM-TRIB2 cells treated with U0126. (E and F) Statistical analysis of STAT3 and p-ERK expression. Data are presented as the mean \pm standard deviation (n=3). ${ }^{*} \mathrm{P}<0.05,{ }^{* * *} \mathrm{P}<0.01$ and ${ }^{* * *} \mathrm{P}<0.001$. TRIB2, Tribble homologue 2.

after treatment with U0126, p-ERK and STAT3 expression was significantly decreased (Fig. 7A-C). These results suggested that expression of the ERK pathway was active in CML K562/ADM cells and U0126 could specifically block this pathway. Furthermore, the expression of p-ERK and STAT3 was clearly downregulated in the K562/ADM-TRIB2 cells. After treatment with U0126, the expression of p-ERK and STAT3 in K562/ADM-TRIB2 cells was significantly decreased, indicating that TRIB2 knockdown may act by blocking ERK pathway activity (Fig. 7D-F). These results suggest that downregulation of TRIB2 affects cell resistance by altering the expression of p-ERK and STAT3 in CML K562/ADM cells.

\section{Discussion}

MDR is a major clinical obstacle for effective tumor chemotherapy, and its impact on chemotherapy in the clinic is worsening. Therefore, novel targeted therapeutic approaches are being explored in order to increase the efficacy of chemotherapy against blood cancers and diseases (14). We observed that TRIB2, MDR1 and MRP1 expression levels were higher in K562/ADM cells, compared with levels in the K562 cells, at both the protein and mRNA levels. This indicated that TRIB2 was involved in the development of MDR in K562/ADM cells.

Numerous studies have shown that the HOX gene family plays an important role in tumor resistance. Knockdown of HOXA5, HOXA10 or HOXB4 was found to reverse multidrug resistance of human CML K562/ADM cells (2,15-17). Related studies have also shown that miR-3142 and miR-146a are overexpressed in K562/ADM cells compared with that noted in K562 cells, which promotes normal cell proliferation and enhanced resistance to ADM in vitro $(18,19)$. Similarly, knockdown of miR-224 and let-7i was shown to reverse the MDR of human CML K562/ADM cells (20). It has been reported that TRIB2 expression is significantly increased in tumor tissues from patients, correlating with the increased phosphorylation of AKT, FOXO3a, MDM2 and C/EBP $\alpha(10,21)$. We found that the knockdown of TRIB2 could decrease MDR1 and MRP1 activity in human CML K562/ADM cells, and also reverse intracellular drug accumulation. In the present study, we first focused on evaluating the association between TRIB2 knockdown and the expression of resistance proteins. Our results indicated that the expression levels of drug-resistant proteins were inhibited by suppressing TRIB2, which reduced the efflux of intracellular ADM and reversed cell resistance. In summary, TRIB2 repression could partially reverse the MDR of K562/ADM cells by inhibiting cellular efflux functions and downregulating the expression levels of MDR1 and MRP1, thus elevating intracellular chemotherapeutic accumulation.

To further investigate the mechanism underlying the role of TRIB2 knockdown in reducing cell resistance, we examined the activity of the ERK pathway. ERK1 and ERK2 constitute the ERK1/2 signal transduction pathway. This is mainly composed of the RAS/RAF/MEK/ERK signal transduction cascade, which can be stimulated by various external stimuli $(22,23)$. Expression of the ERK signal transduction 
pathway and drug efflux proteins in hepatocellular carcinoma, gastric cancer and breast cancer cells is reported to be significantly higher compared with the corresponding controls (24-26). Numerous studies have shown that excessive activation of ERK is positively correlated with the presence of numerous resistant tumors, the mechanism of which may be modulated by the overexpression of resistance-related genes and proteins $(27,28)$. We detected that the expression levels of p-ERK and STAT3 in K562/ADM cells were higher when compared with the levels in K562 cells. Meanwhile, knockdown of TRIB2 in normal K562/ADM cells resulted in the reduced activity of p-ERK and STAT3. To test our hypothesis, the ERK signal transduction pathway inhibitor U0126 was used to downregulate ERK phosphorylation. U0126 is an inhibitor of mitogen-activated protein kinase 1 and 2 (MEK1/2), which blocks phosphorylation and activation of ERK1/2 (29-31). In the present study, western blotting showed that p-ERK was significantly inhibited in K562/ADM cells treated with U0126. This indicated that the gene encoding ERK was involved in the effects of TRIB2 knockdown.

Furthermore, this study investigated the ERK downstream product STAT3, which correlates with cell proliferation (32). STAT3-targeted therapy can reverse drug resistance in CML (33). Our results showed that the activity of STAT3 in K562/ADM cells following TRIB2 knockdown was also lower compared with that noted in the control group. While these initial findings are promising, more comprehensive and detailed studies need to be conducted, including in vivo animal models and more precise ERK1/2 pathway assays. Nevertheless, we demonstrated that decreased expression of resistant proteins is associated with inhibition of the ERK pathway through the knockdown of TRIB2, thereby reversing cell MDR.

\section{Acknowledgements}

The present study was supported by the National Natural Science Foundation (grant no. 31371321), the Shandong Science and Technology Committee (grant nos. ZR2014HQ079, ZR2014HL056 and ZR2013HL003), the Foundation of Shandong Educational Committee (grant nos. J17KA121 and J13LE11) and Young Backbone Teacher Development Support Project of Binzhou Medical University.

\section{Competing interests}

The authors declare no competing interests.

\section{References}

1. Clarke CJ and Holyoake TL: Preclinical approaches in chronic myeloid leukemia: From cells to systems. Exp Hematol 47: 13-23, 2017.

2. Cheng Z, Yang N, Liang W, Yan X, Li L and Pan L: Effect of phosphatase and tensin homology deleted on chromosome 10 (PTEN) gene transfection on reversal of multidrug resistance in K562/ADM cells. Leuk Lymphoma 53: 1383-1389, 2012.

3. Yin $\mathrm{J}$ and Zhang $\mathrm{J}$ : Multidrug resistance-associated protein 1 (MRP1/ABCC1) polymorphism: From discovery to clinical application. Zhong Nan Da Xue Xue Bao Yi Xue Ban 36: 927-938, 2011.

4. Reznicek J, Ceckova M, Ptackova Z, Martinec O, Tupova L, Cerveny L and Staud F: MDR1 and BCRP transporter-mediated drug-drug interaction between rilpivirine and abacavir and effect on intestinal absorption. Antimicrob Agents Chemother 61: e00837-17, 2017.
5. Chen JR, Jia XH, Wang H, Yi YJ, Wang JY and Li YJ: Timosaponin A-III reverses multi-drug resistance in human chronic myelogenous leukemia K562/ADM cells via downregulation of MDR1 and MRP1 expression by inhibiting PI3K/Akt signaling pathway. Int J Oncol 48: 2063-2070, 2016.

6. Liang KL, O'Connor C, Veiga JP, McCarthy TV and Keeshan K TRIB2 regulates normal and stress-induced thymocyte proliferation. Cell Discov 2: 15050, 2016.

7. Gilby DC, Sung HY, Winship PR, Goodeve AC, Reilly JT and Kiss-Toth E: Tribbles-1 and -2 are tumour suppressors, down-regulated in human acute myeloid leukaemia. Immunol Lett 130: $115-124,2010$

8. Yokoyama T and Nakamura T: Tribbles in disease: Signaling pathways important for cellular function and neoplastic transformation. Cancer Sci 102: 1115-1122, 2011.

9. Hannon MM, Lohan F, Erbilgin Y, Sayitoglu M, O'Hagan K, Mills K, Ozbek U and Keeshan K: Elevated TRIB2 with NOTCH1 activation in paediatric/adult T-ALL Br J Haematol 158: 626-634, 2012.

10. Hill R, Madureira PA, Ferreira B, Baptista I, Machado S, Colaco L, Santos M, Liu NS, Dopazo A, Ugurel S, et al: TRIB2 confers resistance to anti-cancer therapy by activating the serine/threonine protein kinase AKT. Nat Commun 8: 14687 , 2017.

11. Chen T, Wang C, Liu Q, Meng Q, Sun H, Huo X, Sun P, Peng J, Liu Z, Yang X and Liu K: Dasatinib reverses the multidrug resistance of breast cancer MCF7 cells to doxorubicin by downregulating P-gp expression via inhibiting the activation of ERK signaling pathway. Cancer Biol Ther 16: 106-114, 2015.

12. Yuan WQ, Zhang RR, Wang J, Ma Y, Li WX, Jiang RW and Cai SH: Asclepiasterol, a novel C21 steroidal glycoside derived from asclepias curassavica, reverses tumor multidrug resistance by down-regulating P-glycoprotein expression. Oncotarget 7: 31466-31483, 2016

13. Sheng Y, You Y and Chen Y: Dual-targeting hybrid peptide-conjugated doxorubicin for drug resistance reversal in breast cancer. Int J Pharm 512: 1-13, 2016.

14. Jacobs RW, Awan FT, Leslie LA, Usmani SZ and Ghosh N: The shrinking role of chemotherapy in the treatment of chronic lymphocytic leukemia. Expert Rev Hematol 9: 1177-1187, 2016.

15. Li N, Jia X, Wang J, Li Y and Xie S: Knockdown of homeobox A5 by small hairpin RNA inhibits proliferation and enhances cytarabine chemosensitivity of acute myeloid leukemia cells. Mol Med Rep 12: 6861-6866, 2015.

16. Wang H, Jia XH, Chen JR, Yi YJ, Wang JY, Li YJ and XIE SY: HOXB4 knockdown reverses multidrug resistance of human myelogenous leukemia K562/ADM cells by downregulating P-gp, MRP1 and BCRP expression via PI3K/Akt signaling pathway. Int J Oncol 49: 2529-2537, 2016.

17. Yi YJ, Jia XH, Wang JY, Li YJ, Wang H and Xie SY: Knockdown of HOXA10 reverses the multidrug resistance of human chronic mylogenous leukemia K562/ADM cells by downregulating P-gp and MRP-1. Int J Mol Med 37: 1405-1411, 2016.

18. Zhao L, Shan Y, Liu B, Li Y and Jia L: Functional screen analysis reveals miR-3142 as central regulator in chemoresistance and proliferation through activation of the PTEN-AKT pathway in CML. Cell Death Dis 8: e2830, 2017.

19. Liu W, He J, Yang Y, Guo Q and Gao F: Upregulating miR-146a by physcion reverses multidrug resistance in human chronic myelogenous leukemia K562/ADM cells. Am J Cancer Res 6: 2547-2560, 2016.

20. Zhou H, Li Y, Liu B, Shan Y, Li Y, Zhao L, Su Z and Jia L: Downregulation of miR-224 and let-7i contribute to cell survival and chemoresistance in chronic myeloid leukemia cells by regulating ST3GAL IV expression. Gene 626: 106-118, 2017.

21. Keeshan K, He Y, Wouters BJ, Shestova O, Xu L, Sai H, Rodriguez CG, Maillard L, Tobias JW, Valk P, et al: Tribbles homolog 2 inactivates $\mathrm{C} / \mathrm{EBP} \alpha$ and causes acute myelogenous leukemia. Cancer Cell 10: 401-411, 2006.

22. McCubrey JA, Steelman LS, Chappell WH, Abrams SL, Wong EW, Chang F, Lehmann B, Terrian DM, Milella M, Tafuri A, et al: Roles of the Raf/MEK/ERK pathway in cell growth, malignant transformation and drug resistance. Biochim Biophys Acta 1773: 1263-1284, 2007.

23. Yang S and Liu G: Targeting the Ras/Raf/MEK/ERK pathway in hepatocellular carcinoma. Oncol Lett 13: 1041-1047, 2017.

24. Guo Y, Ding Y, Zhang T and An H: Sinapine reverses multi-drug resistance in MCF-7/dox cancer cells by downregulating FGFR4/FRS2alpha-ERK1/2 pathway-mediated NF-kappaB activation. Phytomedicine 23: 267-273, 2016. 
25. Buonato JM and Lazzara MJ: ERK1/2 blockade prevents epithelial-mesenchymal transition in lung cancer cells and promotes their sensitivity to EGFR inhibition. Cancer Res 74: 309-319, 2014.

26. Zhao YY, Yu L, Liu BL, He XJ and Zhang BY: Downregulation of P-gp, Ras and p-ERK1/2 contributes to the arsenic trioxide-induced reduction in drug resistance towards doxorubicin in gastric cancer cell lines. Mol Med Rep 12: 7335-7343, 2015.

27. Fan DP, Zhang YM, Hu XC, Li JJ and Zhang W: Activation of AKT/ERK confers non-small cell lung cancer cells resistance to vinorelbine. Int J Clin Exp Pathol 7: 134-143, 2013.

28. Ochi N, Takigawa N, Harada D, Yasugi M, Ichihara E, Hotta K, Tabata M, Tanimoto M and Kiura K: Src mediates ERK reactivation in gefitinib resistance in non-small cell lung cancer. Exp Cell Res 322: 168-177, 2014.

29. Droebner K, Pleschka S, Ludwig S and Planz O: Antiviral activity of the MEK-inhibitor U0126 against pandemic H1N1v and highly pathogenic avian influenza virus in vitro and in vivo. Antiviral Res 92: 195-203, 2011.
30. Shukla V, Coumoul X, Wang RH, Kim HS and Deng CX: RNA interference and inhibition of MEK-ERK signaling prevent abnormal skeletal phenotypes in a mouse model of craniosynostosis. Nat Genet 39: 1145-1150, 2007.

31. Tong Y, Huang $\mathrm{H}$ and Pan H: Inhibition of MEK/ERK activation attenuates autophagy and potentiates pemetrexed-induced activity against HepG2 hepatocellular carcinoma cells. Biochem Biophys Res Commun 456: 86-91, 2015.

32. Gu FM, Li QL, Gao Q, Jiang JH, Huang XY, Pan JF, Fan J and Zhou J: Sorafenib inhibits growth and metastasis of hepatocellular carcinoma by blocking STAT3. World J Gastroenterol 17: 3922-3932, 2011.

33. Gleixner KV, Schneeweiss M, Eisenwort G, Berger D, Herrmann H, Blatt K, Greiner G, Byrgazov K, Hoermann G, Konopleva M, et al: Combined targeting of STAT3 and STAT5: A novel approach to overcome drug resistance in chronic myeloid leukemia. Haematologica 102: 1519-1529, 2017. 